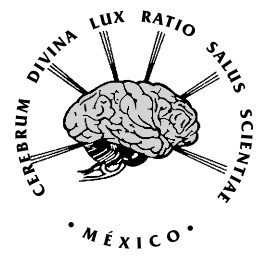

\title{
Efecto del índice enfermera-paciente en la calidad de atención: Estudio realizado en pacientes con enfermedades crónico- degenerativas en el Servicio de Medicina Interna
}

\author{
Effect of contents nurse-patient quality of care: Study in patients \\ with chronic degenerative in internal medicine diseases
}

\author{
Marisol Malvaez Castillo, ${ }^{*}$ Karen Luz Hernández Guerrero, ${ }^{*}$ Nancy Suárez Ortega, ${ }^{*}$ \\ Vania Zuleica Pacheco Sánchez, ${ }^{*}$ Blanca Leticia Rodríguez Madrigal, ${ }^{*}$ \\ Angélica Guadalupe Dávalos-Alcázar**
}

* Licenciada en Enfermería y Obstetricia Universidad Nacional Autónoma de México, Facultad de Estudios Profesionales Zaragoza. UNAM FES Z.

** Maestra en Ciencias de la Educación, Profesor de asignatura A, Universidad Nacional Autónoma de México, UNAM; Facultad de Estudios Superiores Zaragoza FES Z.

\begin{tabular}{|c|c|}
\hline & UMEN \\
\hline $\begin{array}{r}\text { Palabras clave: } \\
\text { Atención, } \\
\text { calidad, crónico- } \\
\text { degenerativa, } \\
\text { cuidados, índice } \\
\text { enfermera- } \\
\text { paciente. }\end{array}$ & $\begin{array}{l}\text { Los indicadores para determinar el número de personal de enfermería necesario deben responder a la demanda epidemiológica que } \\
\text { caracteriza a nuestro país, dominada por enfermedades crónico-degenerativas que requieren un cuidado complejo que garantice } \\
\text { la calidad de atención en salud y reduzca el riesgo de lesiones, maximizando la salud de los pacientes. La demanda de atención } \\
\text { exige a las instituciones realizar la asignación del personal sin considerar la gravedad de la enfermedad, lo que puede alterar la } \\
\text { calidad de atención. El objetivo de este trabajo fue evaluar, en un hospital de segundo nivel, la calidad de atención brindada a } \\
\text { los pacientes con enfermedades crónico-degenerativas en relación al índice enfermera-paciente en medicina interna de atención. } \\
\text { La metodología utilizada fue cuantitativa, longitudinal, prospectiva, no experimental, analítica y correlacional, en } 47 \text { pacientes por } \\
\text { muestra probabilística. Por día se registró el índice real enfermera-paciente, se aplicó una cédula para valorar el estado integral del } \\
\text { paciente a su ingreso y egreso, así como los cuidados de enfermería, utilizando los indicadores de calidad. El análisis se realizó con } \\
\text { el Programa SPSS v20.0, e interpretación correlacional de Pearson y Spearman. Resultados: No se encontró ninguna asociación } \\
\text { significativa (Rho Spearman =0.00) entre la calidad de atención y los pacientes recibidos en el servicio, ni entre la calidad de atención } \\
\text { y el personal promedio que labora en el Servicio de Medicina Interna. Conclusiones: Los indicadores establecidos por la Comisión } \\
\text { Interinstitucional de Enfermería están basados en datos epidemiológicos de 1997, los cuales no responden al estándar de cuidado }\end{array}$ \\
\hline
\end{tabular}

\section{ABSTRACT}

Key words:

Care, quality,

chronic-

degenerative,

care, nurse-

patient ratio.
Indicators to determine the number of nurses needed, must respond to the epidemiological demand that characterizes our country that is dominated by chronic degenerative diseases requiring complex care, to ensure the quality of health care, reduce the risk of injuries, maximizing the health of patients. The demand for care requires institutions make the assignment of staff regardless of disease severity, such a situation may alter the quality of care provided to patients. The objective was to evaluate the quality of care provided to patients with chronic degenerative diseases relating to nurse patient ratio of internal medicine at a hospital in secondary care. The methodology used was quantitative, longitudinal, prospective, non-experimental, analytical and correlational, in 47 patients by probabilistic sample. By day the actual nurse-patient ratio were recorded, 
a charter was applied to assess the overall condition of the patient at admission and discharge, as well as nursing care, using quality indicators. The analysis was performed using SPSS v20.0 program, interpretation and correlation of Pearson and Spearman. Results: No significant association (Spearman Rho $=0.00$ ) was not found between the quality of patient care and service received, or between quality of care and the average staff working in the Department of Internal Medicine. Conclusions: The indicators established by the Inter Nursing Facility, are based on epidemiological data from 1997, which do not respond to standard of care currently required.

\section{INTRODUCCIÓN}

Actualmente, el perfil epidemiológico que caracteriza a nuestro país está dominado por enfermedades crónicodegenerativas. Esta transición va en relación al envejecimiento de la población y al creciente desarrollo de riesgos relacionados con estilos de vida poco saludables. En el 2010, la Organización Mundial de la Salud (OMS) menciona que las enfermedades no transmisibles representan el $63 \%$ de las muertes en el mundo. ${ }^{1}$ De acuerdo con el Instituto Nacional de Estadística y Geografía (INEGI), las enfermedades no transmisibles ocupan las tres principales causas de mortalidad en México, entre ellas las enfermedades isquémicas del corazón, la diabetes mellitus y los tumores malignos. ${ }^{2}$

Estos padecimientos representan las principales causas de mortalidad y morbilidad en México y en el mundo. Aun cuando existen programas para educar a la población sobre la prevención de tales enfermedades y sus complicaciones, es nuestra corresponsabilidad, como parte del personal de salud, hacer frente a las demandas de atención de la población; por ello se crearon los indicadores de calidad de los servicios de enfermería, para garantizar la calidad de los servicios de salud.

La OMS define a la calidad de la asistencia sanitaria como: "asegurar que cada paciente reciba el conjunto de servicios diagnósticos y terapéuticos más adecuados para conseguir una atención sanitaria óptima, teniendo en cuenta todos los factores y conocimientos del paciente así como del servicio médico, y lograr el mejor resultado con el mínimo riesgo de efectos iatrogénicos y la máxima satisfacción del paciente con el proceso". ${ }^{3}$

Al hablar de garantía y mejora de la calidad de los servicios de enfermería se debe prestar importancia a la relación que tienen estas características con los recursos humanos; por ejemplo, a nivel nacional se cuenta con 2.2 enfermeras por cada 1,000 habitantes, cifra muy inferior al promedio de los países de la OCDE (Organización para la Cooperación y el Desarrollo Económicos). ${ }^{4}$

En el 2003, la Subsecretaría de Innovación y Calidad, en conjunto con la Comisión Interinstitucional de Enfermería, ${ }^{5}$ crearon una guía técnica para la dotación de recursos humanos con el objetivo de que las instituciones de salud cuenten con lineamientos para calcular los recursos humanos en enfermería adecuados para cada servicio.

Queda entonces responder a la pregunta: ¿Realmente se da calidad de atención teniendo un índice enfermerapaciente reducido? En un estudio realizado por Aiken L. y colaboradores, ${ }^{6}$ sobre "Satisfation with inpatient acquiered inmunodeficiency sindrome care: a national comparison of dedicated and scattered-bed units" cuyo objetivo fue determinar la asociación entre la relación paciente-enfermera con la mortalidad de los pacientes, los resultados obtenidos fueron que con cada paciente adicional a una enfermera que ya se encargase de cuatro aumentaba un $7 \%$ la probabilidad de muerte dentro de los 30 días siguientes a la admisión y aumentaba también un $7 \%$ la probabilidad de fracaso terapéutico.

En otro estudio de Aiken, Kesselring y Schubert, ${ }^{7}$ realizado en el 2003 y titulado "Job satisfaction and treatment outcomes support is vital for nursing quality" se constata la asociación que existe entre las buenas condiciones laborales como soporte vital para un cuidado de enfermería con calidad; se afirma que al aumentar la carga laboral de cuatro pacientes a seis se incrementan en un $14 \%$ las probabilidades de que esos pacientes mueran dentro de los 30 días siguientes a su admisión, y si la carga de trabajo se eleva a ocho pacientes, la mortalidad crece un $31 \%$. Por otro lado, cuando se aumenta el número de enfermeras se reduce el número de infecciones del tracto urinario, y los índices de "fracaso terapéutico" son menores. ${ }^{8}$

Mesa L., en su estudio "La enfermera en la primera línea de defensa en la seguridad del paciente", ha demostrado el impacto que se tiene al contar con un número de enfermeras adecuado, ya que disminuye la mortalidad de los pacientes, hay menor índice de infecciones nosocomiales, úlceras por presión y complicaciones postoperatorias, estancia hospitalaria más corta, mejor calidad de la atención, mayor seguridad del paciente y mayor satisfacción del usuario. ${ }^{9}$

No se encontraron datos o antecedentes de investigaciones en México sobre este tema tomando en cuenta los indicadores de salud de los Servicios de Enfermería; por ello, el propósito del estudio es analizar cómo se relaciona el índice enfermera-paciente real en la calidad de atención de los pacientes con enfermedades crónico-degenerativas en el Servicio de Medicina Interna de un Hospital de Segundo Nivel de Atención.

\section{MATERIAL Y MÉTODOS}

Es una investigación de tipo: no experimental, cuantitativa, longitudinal, prospectiva, analítica y correlacional.

Se realizó en pacientes con enfermedades crónico-degenerativas del Servicio de Medicina Interna del Hospital General de Zona No. 1-A "Venados" del IMSS, extrayén- 
dose una muestra de la población de tipo probabilística, del $30 \%$ con un nivel de error del $5 \%$ y nivel de confianza del $95 \%$ para que la aplicación de los criterios de eliminación no altere la muestra.

Se analizaron 47 pacientes diagnosticados con enfermedades crónico-degenerativas sin distinción de género ni edad, que quisieran participar, previa autorización del consentimiento informado y que ingresaron al servicio de medicina interna a partir del 7 de mayo, y para su análisis el 21 y 22 de mayo del 2012.

La valoración de los pacientes se realizó por medio de la aplicación de una herramienta específica para determinar la calidad de atención que recibió el paciente desde el momento de su ingreso hasta el día de su alta o hasta el 18 de mayo, fecha en la que concluyó la recolección de datos.

El instrumento utilizado para valorar la calidad de atención de los Servicios de Enfermería fueron los indicadores de calidad establecidos por la Secretaría de Salud, agregando así otros tres (lavado de manos, aspiración de secreciones traqueo-bronquiales y vigilancia y control del proceso de cambio de bolsa de diálisis peritoneal) ${ }^{10}$ cuya cédula de valoración fue tomada del Manual de Evaluación de la calidad del Servicio de Enfermería de Ortega y Suárez (2009) del Instituto Nacional de Cardiología "Ignacio Chávez". También se observó la evolución de los pacientes previamente seleccionados; dicha observación duró cuatro días.

Se evaluó también el grado académico o título académico al que pertenece el personal de enfermería del Servicio de Medicina Interna y por día se registró el índice real enfermera-paciente en dicho servicio.

Todos los datos se concentraron y analizaron con el Programa SPSS versión 20; las variables se correlacionaron con interpretación de Spearman y $r$ de Pearson.

\section{RESULTADOS}

Del total de 47 pacientes, $49 \%$ (23) eran hombres y el $51 \%$ (24) mujeres; respecto a la edad, la media fue de 63 años, encontrándose una mínima de 19 y una máxima de 92; las enfermedades que más predominaron fueron insuficiencia renal crónica (47\%), hipertensión arterial sistémica (HTA) (19\%), diabetes mellitus (DM) (17\%) y otras con el $19 \%$ [síndrome de inmunodeficiencia adquirida (SIDA), enfermedad pulmonar obstructiva crónica (EPOC), insuficiencia cardiaca congestiva (ICC), entre las más relevantes], siendo más frecuentes en hombres con un $55 \%$ (33 padecimientos) que en mujeres con un $45 \%$ (27 padecimientos).

El $70 \%$ de los pacientes estuvo en promedio ocho días hospitalizado; de acuerdo con las complicaciones derivadas de la hospitalización, el 60\% (28 pacientes) no mostró ninguna complicación, el 23\% (11 pacientes) presentó úlceras por presión; el $6 \%$ sufrió caídas, el $6 \%$ padeció algún tipo de infección en herida o catéter y sólo $2 \%$ presentó neumonía. Los pacientes con más complicaciones derivadas de la hospitalización fueron los de mayor edad, y quienes tenían más de tres días de estancia hospitalaria; el porcentaje de mujeres fue mayor que el de hombres.
Respecto al personal de enfermería, se encontró que el $96 \%$ cuenta con grado de estudios profesionales; la relación enfermera general y auxiliar es uno a uno.

De los nueve indicadores de calidad evaluados entre los Servicios de Enfermería, tomados de la Comisión Interinstitucional de Enfermería y del Instituto Nacional de Cardiología, se encontró lo siguiente: más del $70 \%$ del personal cumple con los indicadores de trato digno, lavado de manos, prevención de infecciones de vías urinarias en pacientes con sonda vesical, prevención de úlceras por presión y aspiración de secreciones.

Mientras que al menos el 50\% cumplió con el indicador de prevención de caídas, ministración de medicamentos por vía oral, vigilancia y control de proceso de cambio de bolsa de diálisis peritoneal, menos del $50 \%$ cumplió con el indicador de vigilancia y control de venoclisis instalada. Respecto al tipo de alta, el 90\% fue por mejoría, encontrándose también un nivel de defunciones de $0.45 \%$ durante el mes de mayo.

Se logró cumplir con nuestro objetivo, que era evaluar la calidad de los Servicios de Enfermería con base en los indicadores de calidad; sin embargo, nuestra hipótesis no se cumple, ya que no existe correlación alguna entre las variables (Rho Spearman $=0.00$ ) (Figura 1 ) de calidad de atención y número de pacientes recibidos en el servicio, y entre la calidad de atención y el número de personal real que hay en el Servicio de Medicina Interna. Por lo tanto, no influye que a mayor número de pacientes asignados a una enfermera disminuya la calidad de atención.

\section{DISCUSIÓN Y CONCLUSIONES}

Consideramos que los indicadores para la distribución del recurso humano no corresponden a la demanda de aten-

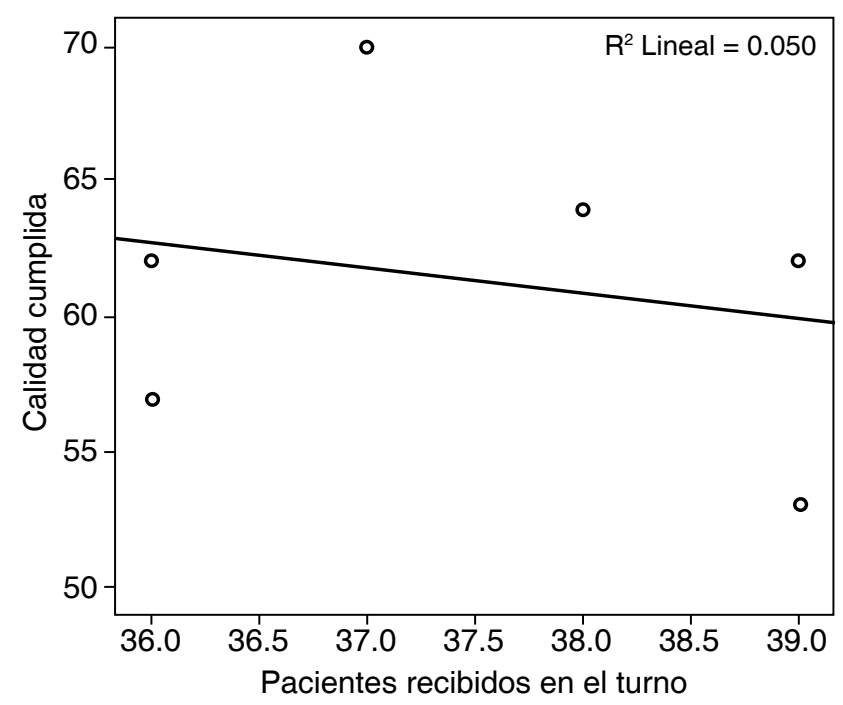

Fuente: Encuesta realizada en el Servicio de Medicina Interna del Hospital General de Zona No. 1-A, Mayo 2012.

Figura 1. Calidad de atención en relación al número de pacientes. 
Enf Neurol (Mex) • 2014 • Vol. 13• Núm. 3 • 118-121

ción solicitada, debido a que actualmente hay un aumento constante en el número de personas que presentan una enfermedad crónico-degenerativa, las cuales acuden a los servicios médicos principalmente ante complicaciones derivadas de las mismas, no al inicio, lo que hace que la atención tenga que ser de mayor complejidad y requiera más tiempo y recurso humano para cubrir dicha demanda; por lo tanto, los indicadores para establecer el número de personal de enfermería requerido en un servicio establecido por la Comisión Interinstitucional no cumplen, pues se basan en datos epidemiológicos de 1997, y no en la situación epidemiológica actual en relación a la pirámide poblacional y tasas de morbilidad y mortalidad actualizadas.

\section{BIBLIOGRAFÍA}

1. Organización Mundial de la Salud. Informe Mundial de la OMS. La OMS traza el mapa de las enfermedades no transmisibles en todos los países. Ginebra. OMS; 2011. [Sitio en Internet] [consultado el 26 marzo de 2012], Disponible en: http://www.who.int/mediacentre/news/releases/2011/NCDs_ profiles_20110914/es/index.html

2. Secretaría de Salud. Programa Nacional de Salud 20072012. México: 2007. p. 29.

3. Varo J. Gestión estratégica de la calidad en los servicios sanitarios: un modelo de gestión hospitalaria. Madrid: Ediciones Díaz de Santos; 1994. pp. 60-61.
4. Organización para la Cooperación y el Desarrollo Económico. Estudios de la OCDE sobre los Sistemas de Salud México. París, 2005. p. 33.

5. Secretaría de Salud, Subsecretaría de Innovación y Calidad, Comisión Interinstitucional de Enfermería. Guía técnica para la dotación de recursos Humanos en Enfermería. México 2003. [Sitio en Internet] [consultado 18 abril 2012]. Disponible en: http://ecaths1.s3.amazonaws.com/sotogestion/Recurso\%20humano.Guia\%20dotacion\%20personal.\%20Mx.pdf

6. Aiken LH, Clarke SP, Sloane DM, Sochalski J, Silber JH. Satisfaction with inpatient acquired immunodeficiency syndrome care: a national comparison of dedicated and scattered-bed units. JAMA. 2002; 16: 1-16.

7. Gaviria ND. La evaluación del cuidado de enfermería: un compromiso de disciplina. REDALIC. Colombia. 2009; 23: 24-33.

8. Clarke SP, Sloane DM, Aiken LH. Effects of hospital staffing and organizational climate on needlestick injuries to nurses. Am J Public Health. 2002; 92: 1115-1119.

9. Mesa L. La enfermera es la primera línea de defensa en la seguridad del paciente. Evidentia [Sitio en Internet] 2007; 4 (18). Disponible en: http://www.index-f.com/evidentia/n18/ r391articulo.php

10. Ortega MV, Suárez VG. Manual de evaluación de la calidad del servicio de enfermería: estrategias para su aplicación. $2^{\underline{a}}$ ed. México: Médica Panamericana; 2009.

Dirección para Correspondencia:

Marisol Malvaez Castillo.

Calle Progreso Núm. 12, San Lorenzo Tezonco, 09900, Iztapalapa,

México, D.F. E-mail: mary_solmmc@ @otmail.com 